\title{
Immunohistochemical analysis of in vivo UVB-induced secretion of IL-1 $\alpha$ and IL-6 in keratinocytes
}

\author{
HIROMI NARUMI, HAJIME NAKANO, YASUSHI MATSUZAKI, DAISUKE SAWAMURA and KATUMI HANADA
}

Department of Dermatology, Hirosaki University Graduate School of Medicine, Hirosaki, Aomori 036-8562, Japan

Received December 20, 2010; Accepted March 22, 2011

DOI: $10.3892 / \mathrm{mmr} .2011 .478$

\begin{abstract}
Keratinocytes play an important role in the inflammatory response of the skin. UVB stimulates keratinocytes to secrete several cytokines. To our knowledge, there are no reports that have examined which layer or cell type is responsible for producing these cytokines. Thus, in this study we performed immunohistochemical experiments to determine the potential cells or layers that express interleukin (IL)-1 and IL-6. The results show that the expression of IL- $1 \alpha$ and IL-6 was induced 3 and $6 \mathrm{~h}$ after irradiation, respectively. Furthermore, IL-1 $\alpha$ was maximally expressed earlier than IL-6, suggesting that IL-1 $\alpha$ exists in the cytokine cascade of the UVB response upstream of IL-6. In addition, IL-1 $\alpha$ expression began in the upper layers of the epidermis, whereas all layers expressed IL-6. This study provides further insight into the roles of cytokines in UVB-induced skin inflammation.
\end{abstract}

\section{Introduction}

The epidermis is the outermost layer of the skin. The majority of its cells are keratinocytes, which comprise $95 \%$ of the total cell number. Keratinocytes play an essential role in the inflammatory response of the skin and produce many factors due to several exogenous stimuli, including UVB irradiation (1-3). Some of the factors produced due to UVB stimulation are cytokines, including interleukin (IL)-1 $\alpha$ IL-8, IL-6, Il-10 and tumor necrosis factor (TNF)- $\alpha$. The secretion of these cytokines by keratinocytes triggers a cutaneous inflammatory response that develops in skin exposed to UVB (4-6).

Keratinocytes move from the basement membrane zone towards the skin surface, forming several well-defined layers during transit. Those layers in ascending order are the basal, spinous, granular and cornified layers. While UVB is well known to induce the expression of proinflammatory cytokines, there are no available reports on which layer or cell type is

Correspondence to: Dr Hiromi Narumi, Department of Dermatology, Hirosaki University Graduate School of Medicine, 5 Zaifu-cho, Hirosaki, Aomori 036-8562, Japan

E-mail: derma@cc.hirosaki-u.ac.jp

Key words: sunburn, cytokine, epidermis, ultraviolet right responsible for their production. Therefore, we performed immunohistochemical experiments to determine which cells or layers express IL-1 and IL-6.

\section{Materials and methods}

Hirosaki hairless rats. The Hirosaki hairless rat is a mutant strain spontaneously derived from the Sprague-Dawley rat; the inheritance of the hairlessness trait is autosomal recessive (7). Hirosaki hairless rats were housed in plastic cages in airconditioned rooms with a 12-h light/dark cycle at the Institute for Animal Experiments of Hirosaki University Graduate School of Medicine, and had free access to water and food. This study was carried out in accordance with the Guidelines for Animal Experimentation of Hirosaki University.

$U V B$ radiation source. As a UVB source, a bank of 7 fluorescent sunlamps (FL20SE.30; Toshiba Medical Supply, Tokyo, Japan) emitting rays of 275-305 $\mathrm{nm}$ with a peak at $305 \mathrm{~nm}$ was used $(8,9)$. The radiation dose was measured using a radiometer (UVR-3036/S; Clinical Supply, Japan).

Experimental design and $U V$ irradiation. The preliminary data showed that the minimal erythema dose of the Hirosaki hairless rat is $100 \mathrm{~mJ} \cdot \mathrm{cm}^{-2}$. A single dose of 2 minimal erythema UVB doses $\left(200 \mathrm{~mJ} \cdot \mathrm{cm}^{-2}\right)$ was administered to the dorsal sides of the Hirosaki hairless rats. The rat skins were excised at various time points after irradiation, and subjected to immunohistochemistry.

Immunohistochemistry. Skin tissues from treated rats were fixed in $10 \%$ formaldehyde and embedded in paraffin. Immunohistochemical staining for IL-1 $\alpha$ and IL-6 was performed using the avidin-biotin-peroxidase complex (ABC) method (10) with their respective antibodies (Santa Cruz Biotechnology, Santa Cruz, CA). The biotinylated secondary antibody and Vectastain $\mathrm{ABC}$ kit were obtained from Vector Laboratories (Burlingame, CA). Specific binding was visualized with a 3,3'-diaminobenzidine tetrahydrochloride solution. Sections were then lightly counterstained with hematoxylin for microscopic examination. The specimens were examined and photographed using a microscope (Coolscope; Nikon, Tokyo, Japan) interfaced with a computer. The semiquantification of immunoreactivity on each slide was evaluated using NIH Image J software. The expression levels are expressed as mean (SD). 


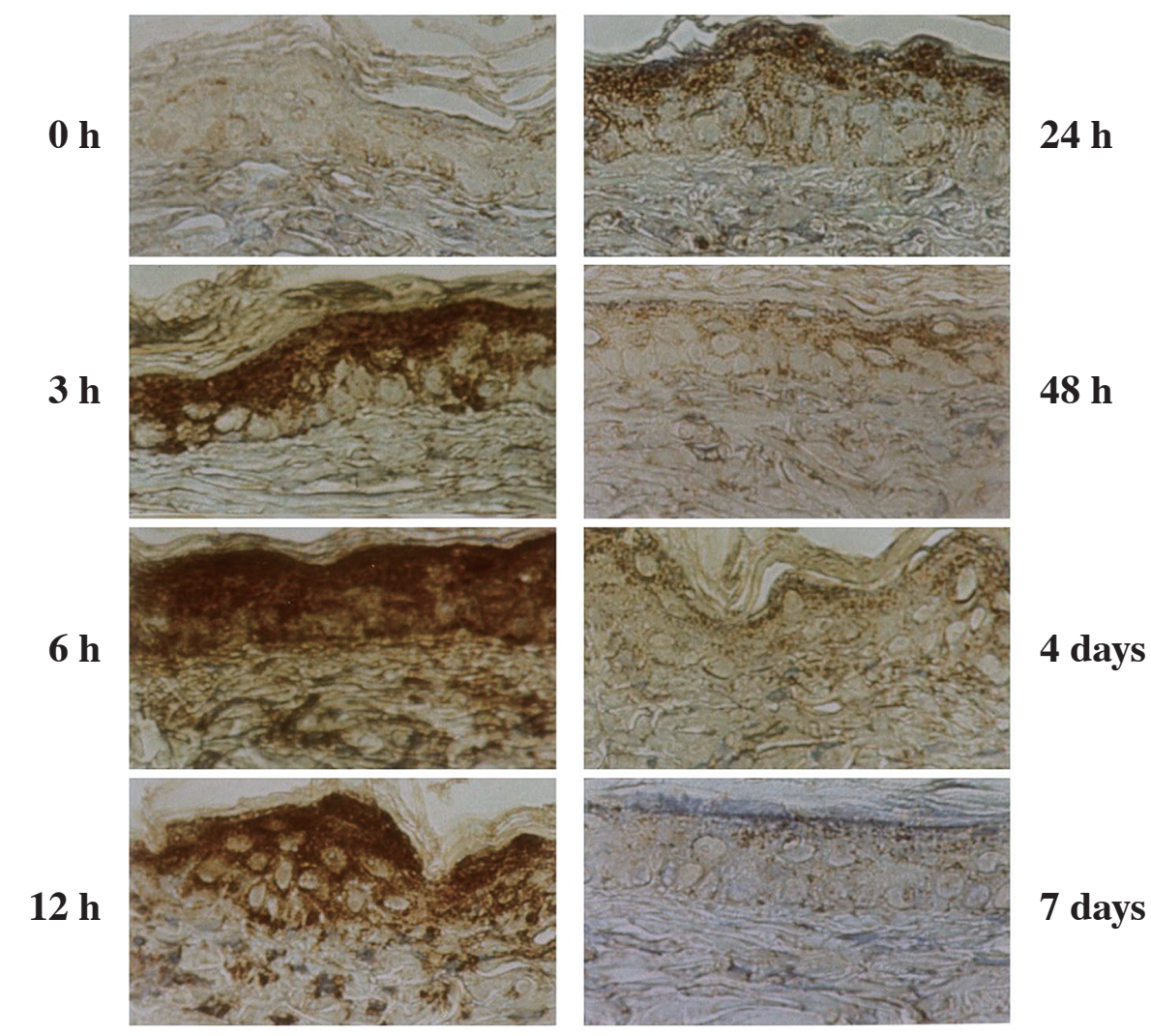

Figure 1. UVB-induced IL-1 $\alpha$ expression. Hirosaki hairless rats were treated with a single administration of 2 minimal erythema doses and skin biopsies were collected at 3, 6, 12, 24 and $48 \mathrm{~h}$, and 4 and 7 days after irradiation. IL-1 $\alpha$ expression was examined by immunohistochemistry.

$\mathbf{0 ~ h}$
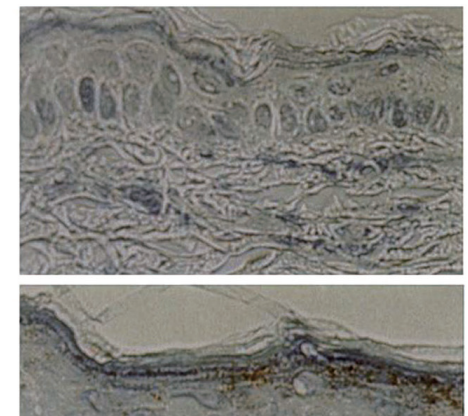

$3 \mathbf{h}$

$6 \mathbf{h}$

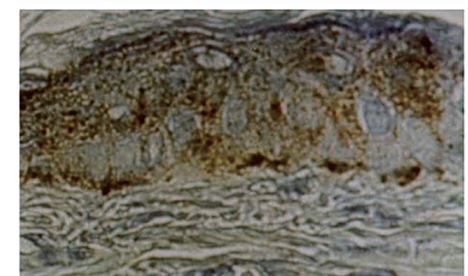

$12 \mathrm{~h}$

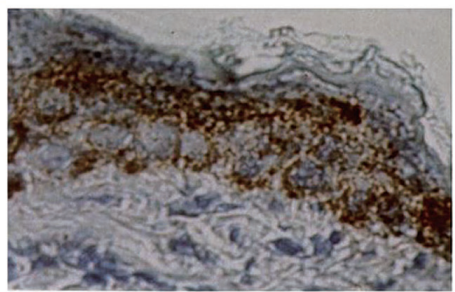

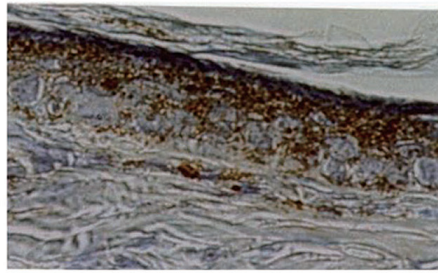
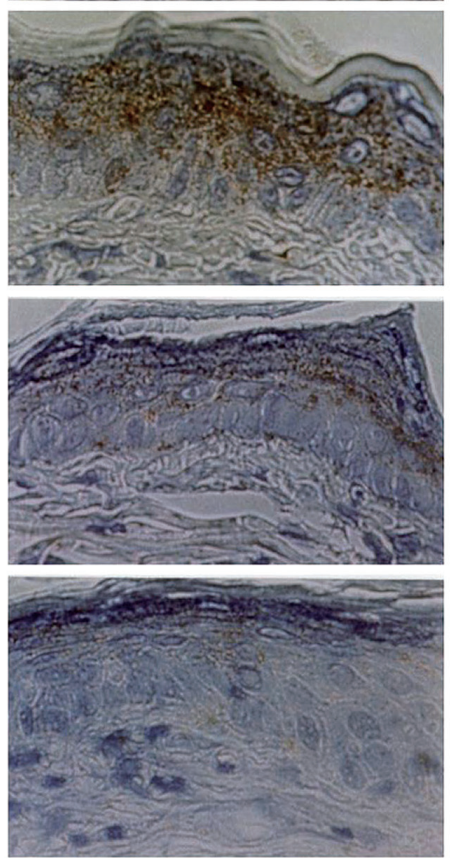

$24 \mathrm{~h}$

$48 \mathrm{~h}$

4 days

7 days

Figure 2. UVB-induced IL-6 expression. Hirosaki hairless rats were treated with a single administration of 2 minimal erythema doses and skin biopsies were collected at 3,6,12, 24 and $48 \mathrm{~h}$, and 4 and 7 days after irradiation. IL-6 expression was examined by immunohistochemistry. 
A

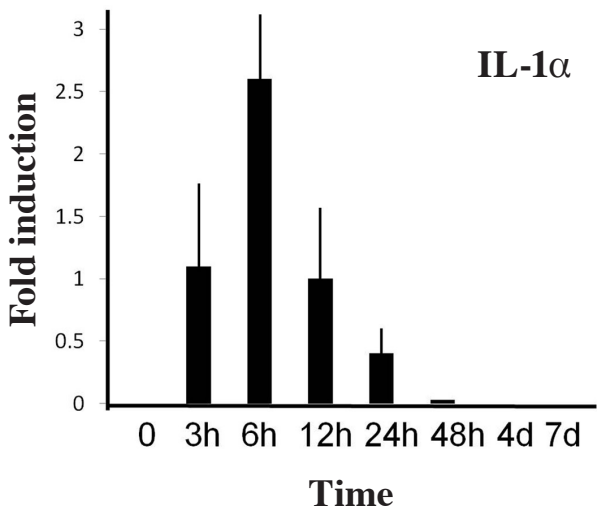

B

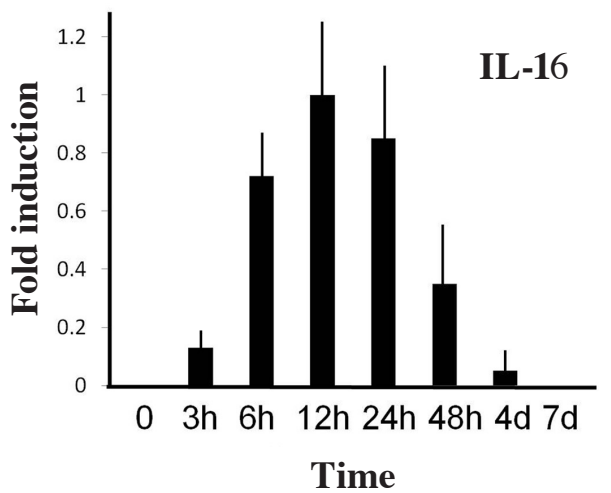

Figure 3. Semiquantification of IL-1 $\alpha$ and IL-6 expression. Hirosaki hairless rats were treated with a single administration of 2 minimal erythema doses and skin biopsies were collected at various times after irradiation. After immunohistochemistry for IL- $1 \alpha$ and IL-6, the semiquantification of immunoreactivity was evaluated using NIH Image J. The expression levels are expressed as the mean (SD).

\section{Results}

The Hirosaki hairless rats were treated with a single administration of 2 minimal erythema UVB doses, then their skin was biopsied at various times after irradiation, and the expression of IL-1 $\alpha$ and IL-6 was immunohistochemically examined (Fig. 1).

IL-1 $\alpha$ expression began in the upper layers of the epidermis $3 \mathrm{~h}$ after UVB irradiation, and was detected in all layers $6 \mathrm{~h}$ after treatment. Subsequently, the immunoreactivity of IL- $1 \alpha$ gradually decreased. Little or no IL-1 $\alpha$ expression was observed $48 \mathrm{~h}$ after irradiation, and no reactivity was detected in any specimen after 4 or 7 days. The semiquantification of IL- $1 \alpha$ reactivity also revealed the maximum expression at $6 \mathrm{~h}$ after treatment (Fig. 3A).

IL-6 expression was detected in the first $6 \mathrm{~h}$ after the UVB treatment, peaking at 12 or $24 \mathrm{~h}$ (Fig. 2). IL-6 expression was detected in almost all layers of the epidermis and completely disappeared 7 days after irradiation. The semiquantification of immunoreactivity revealed that there were no significant differences in expression between $12 \mathrm{~h}$ and $24 \mathrm{~h}$. (Fig. 3B).

\section{Discussion}

In this study, Hirosaki hairless rats were used for all experiments. A recent study shows that an $80-\mathrm{kb}$ section of genomic DNA in $7 \mathrm{q} 36$ containing the basic hair keratin genes $\mathrm{Kb} 21$, $\mathrm{Kb} 23, \mathrm{~Kb} 26$ and $\mathrm{Krt} 2-25$ is deleted in rats (11). Furthermore, abnormalities in keratin genes may cause hairless phenotypes (11). Our group has performed gene transfer experiments and obtained appropriate results (12). When regular mice or rats are used for irradiation experiments, their hair must be shaved. Thus, Hirosaki hairless rats are useful for irradiation experiments because they do not require shaving.

IL-1 $\alpha$ has many physiological functions in immune, metabolic and hematopoietic systems. Furthermore, keratinocytes are a major source of IL-1 $\alpha$. As a proinflammatory cytokine, $\mathrm{IL}-1 \alpha$ is involved in inflammatory and allergic skin diseases such as psoriasis and contact dermatitis. It also plays a key role in many recently defined auto-inflammatory diseases (13). These diseases are characterized by 'sterile' inflammation without infection, or the presence of auto-antibodies or autoreactive T-cells. Reduction in IL-1 activity results in the rapid remission of symptoms. Of note, several auto-inflammatory diseases also affect the skin. IL-6 is also an interleukin that acts as both a pro- and anti-inflammatory cytokine. It is secreted by keratinocytes and stimulates immune response in both normal and abnormal skin conditions (14). The anti-IL-6 antibody tocilizumab is reported to be effective for treating psoriasis, reumatoid arthritis and Castleman's disease (15).

UVB irradiation induces sunburn as well as IL-1 $\alpha$ and IL-6 expression; therefore, these cytokines are responsible for inflammation during sunburn. In this study, we performed immunohistochemical experiments to determine the cells that express IL- $1 \alpha$ and IL-6. We confirmed that the expression of IL- $1 \alpha$ and IL- 6 was induced, detected at 3 and $6 \mathrm{~h}$ after irradiation, respectively. Furthermore, IL- $1 \alpha$ is maximally expressed earlier than IL-6, suggesting that IL-1 $\alpha$ exists in the cytokine cascade of the UVB response upstream of IL-6. In addition, $\mathrm{IL}-1 \alpha$ expression began in the upper layers of the epidermis, whereas all layers expressed IL-6. The lower layers of the epidermis, including the basal layer, which maintains cell division, may possess some protective mechanisms against UVB in contrast with the non-proliferating upper layers.

This study revealed the detailed expression patterns of IL-1 $\alpha$ and IL- 6 in the epidermis and provides further insight intio the roles of cytokines in UVB-induced inflammation. However, we only irradiated the rats with a single administration of 2 minimal erythema doses. Further experiments using different animals and various UVB doses are required. In addition, animals should be repeatedly exposed to UVB in order to understand chronic cutaneous reactions to UVB.

\section{Discussion}

The authors would like to thank Ms. Yuka Toyomaki, Mrs. Yukiko Tamura, Mrs. Yuriko Talagi and Ms. Nanako Seitoh for their excellent technical assistance. This study was supported in part by Grants-in-Aid from the Ministry of Education, Science, Sports and Culture of Japan.

\section{References}

1. Torii K, Maeda A, Saito C, et al: UVB wavelength dependency of antimicrobial peptide induction for innate immunity in normal human keratinocytes. J Dermatol Sci 56: 217-219, 2009.

2. Stork CJ, Martorano LM and Li YV: UVB radiation induces an increase in intracellular zinc in human epidermal keratinocytes. Int J Mol Med 26: 463-469, 2010. 
3. Kovacs D, Raffa S, Flori E, et al: Keratinocyte growth factor down-regulates intracellular ROS production induced by UVB. J Dermatol Sci 54: 106-113, 2009.

4. Kupper TS, Chua AO, Flood P, McGuire J and Gubler U: Interleukin 1 gene expression in cultured human keratinocytes is augmented by ultraviolet irradiation. J Clin Invest 80: 430-436, 1987.

5. Kondo S, Kono T, Sauder DN and McKenzie RC: IL-8 gene expression and production in human keratinocytes and their modulation by UVB. J Invest Dermatol 10: 690-694, 1993.

6. Strickland FM, Darvill A, Albersheim P, et al: Inhibition of UV-induced immune suppression and interleukin-10 production by plant oligosaccharides and polysaccharides. Photochem Photobiol 69: 141-147, 1999.

7. Hanada K, Chiyoya S, Suzuki K, Hashimoto I and Hatayama I: Study of the skin of a new hairless rat mutant. J Dermatol 15 257-262, 1988.

8. Hanada K: Photoprotective role of metallothionein in UV-injury. Metallothionein-null mouse exhibits reduced tolerance agains ultraviolet-B. J Dermatol Sci 23 (Suppl 1): S51-S56, 2000.

9. Hanada K, Sawamura D, Tamai K, Hashimoto I and Kobayashi S: Photoprotective effect of esterified glutathione against ultraviolet B-induced sunburn cell formation in the hairless mice. J Invest Dermatol 108: 727-730, 1997.
10. Kitamura H, Matsuzaki Y, Kimura K, et al: Cytokine modulation of retinoic acid-inducible gene-I (RIG-I) expression in human epidermal keratinocytes. J Dermatol Sci 45: 127-134, 2007.

11. Nanashima N, Akita M, Yamada T, et al: The hairless phenotype of the Hirosaki hairless rat is due to the deletion of an $80-\mathrm{kb}$ genomic DNA containing five basic keratin genes. J Biol Chem 283: 16868-16875, 2008.

12. Meng X, Sawamura D, Tamai K, et al: Keratinocyte gene therapy for systemic diseases. Circulating interleukin 10 released from gene-transferred keratinocytes inhibits contact hypersensitivity at distant areas of the skin. J Clin Invest 101: 1462-1467, 1998.

13. Goldbach-Mansky R and Kastner DL: Autoinflammation: the prominent role of IL-1 in monogenic autoinflammatory diseases and implications for common illnesses. J Allergy Clin Immunol 124: 1141-1149, 2009.

14. Sawamura D, Meng X, Ina S, et al: Induction of keratinocyte proliferation and lymphocytic infiltration by in vivo introduction of the IL-6 gene into keratinocytes and possibility of keratinocyte gene therapy for inflammatory skin diseases using IL-6 mutant genes. J Immunol 161: 5633-5639, 1998.

15. Patel AM and Moreland LW: Interleukin-6 inhibition for treatment of rheumatoid arthritis: A review of tocilizumab therapy. Drug Des Devel Ther 4: 263-278, 2010. 